\title{
Mesures pluviométriques
}

\author{
par P. Viton et J.-C.. Tuifiigo \\ Météorologie Nationale \\ Centre Technique et du Matériel
}

Les nombreuses études et divers projets entrepris par la Météorologie Nationale, en vue d'assurer le remplacement des instruments en service qui ne présentaient pas toutes les caractéristiques souhaitées ont abouti à la construction de deux nouveaux pluviographes. Ces équipements répondent à des besoins bien distincts; le premier composé d'un pluviomètre transducteur d'impulsions (figure 1) et d'un chronototalisateur (figure 2), doit dès 1973, équiper les stations météorologiques de base. Il offre de multiples possibilités : enregistrement à distance, mesure de l'équivalent en eau des précipitations neigeuses, appréciation de l'intensité avec une bonne approximation...

La deuxième, encore au stade de prototype est un appareil plus conventionnel avec enregistrement dans le corps du pluviographe (figure 3).

Il est destiné à des météorologistes pour établir des réseaux d'appoint et aussi à des usagers divers : agronomes, hydrologues, urbanistes, etc., sa caractéristique essentielle tient dans son prix de revient peu élevé.

Le premier problème à résoudre, lors de la mise au point đu pluviomètre transducteur d'impulsions a été celui de sa forme. Il s'agissait de lui donner un encombrement et une forme minimisant les effets du vent sur la captation. A la suite d'une étude en soufflerie sur la répartition des filets d'air à proximité de divers pluviomètres nous avions remarqué l'existence d'une zone d'écoulement rapide au-dessus des bagues des appareils ayant des formes cylindriques ou

N.D.L.R. - Cette étude fait suite à une réunion de la Commission "Hydrologie » du Conseil Supérieur de la Météorologie, au cours de laquelle les problèmes relatifs aux mesures pluviométriques ont été passés en revue. cylindro-côniques (figures $4 a$ et $4 b$ ). Ainsi les précipitations pénétrant dans cette zone sont entraînées par le flux et ne peuvent atteindre le cône récepteur.

Par contre, le pluviomètre dont la silhouette est constituée par un cône (ou tronc de cône) reposant sur un pied support de faible encombrement (figure $4 c$ ) avait l'avantage de moins perturber les filets d'air au-dessus du plan passant par la bague et par conséquent d'affecter moins que les précédents les trajectoires des gouttes et la collecte de cellesci. Enfin, des essais comparatifs ont montré, qu'à Trappes et à l'échelon de l'année, les différences sur les quantités d'eau recueillies par deux pluviomètres, l'un de type $b$, l'autre de type $c$ atteignaient $5 \%$ au profit du type $c$. En conséquence, nous avons été conduits à adapter pour le nouveau pluviographe une forme qui rappelle celle d'un «verre à pied».

Pour assurer la mesure de l'eau recueillie par le cône, nous avons opté pour un compteur volumétrique de type " à augets basculeurs ». Ce compteur simple et peu coûteux, qui fonctionne bien sans certains soins particuliers est pourtant l'objet de vives critiques; on lui reproche en particulier une réponse nettement déficitaire dès que les débits dépassent $165 \mathrm{~cm}^{3} / \mathrm{mn}$. Ce défaut tient à ce que les basculements du système ne sont pas instantanés; entre l'instant où il commence à basculer et l'instant où il a tourné d'un angle suffisant pour la mise en service de l'auget vide, l'auget plein reçoit une quantité d'eau supplémentaire, non prise en compte, qui peut atteindre, pour des débits de $400 \mathrm{~cm}^{3} / \mathrm{mn}$ $15 \%$ de sa capacité nominale. Grâce à deux déflecteurs mobiles (figure 5) sur lesquels s'écoule l'eau provenant du cône récepteur, il a été possible d'utiliser l'énergie cinétique des précipitations pour faire démarrer le basculement du système un peu avant que l'auget n'ait reçu sa charge nominale. 


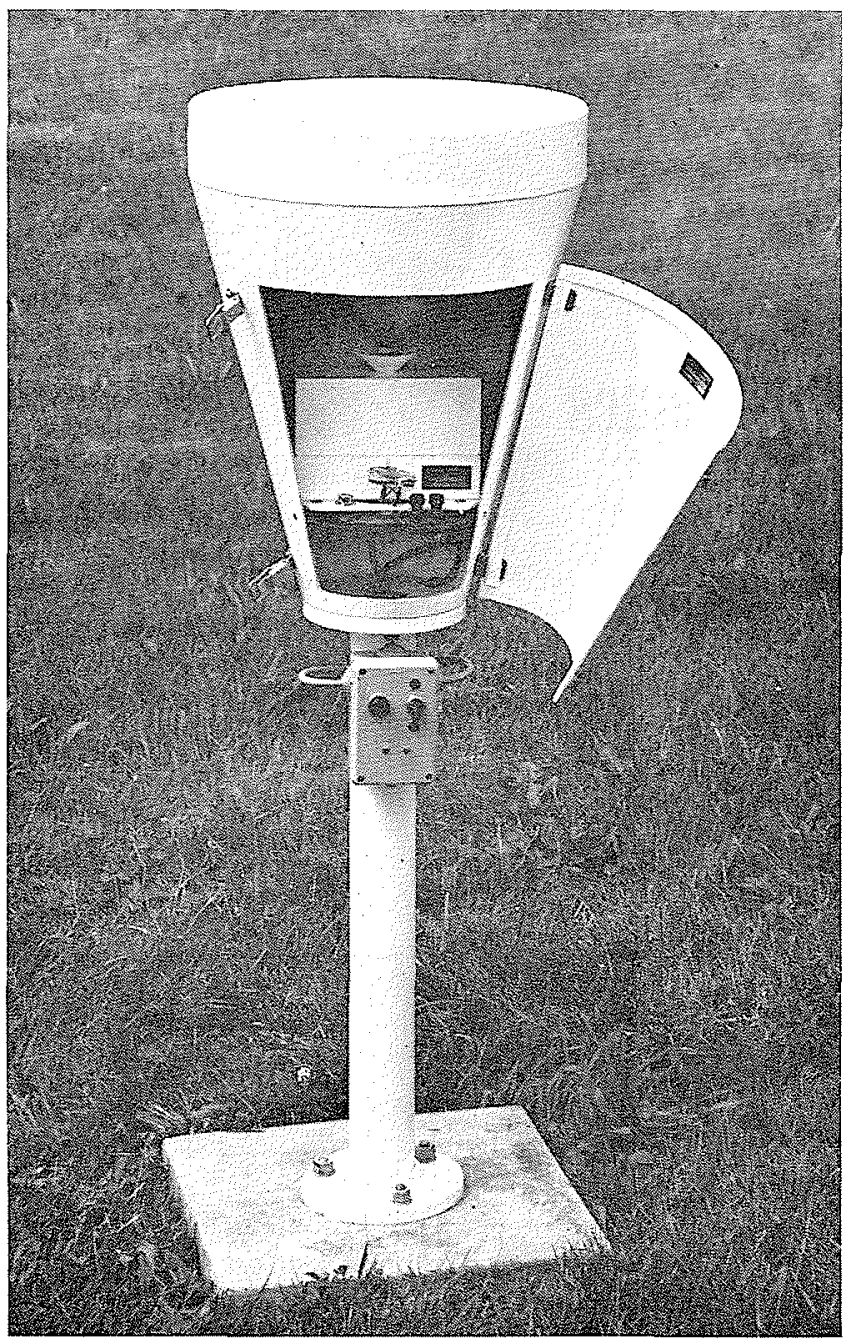

1/ Pluviomètre transducteur d'impulsions.

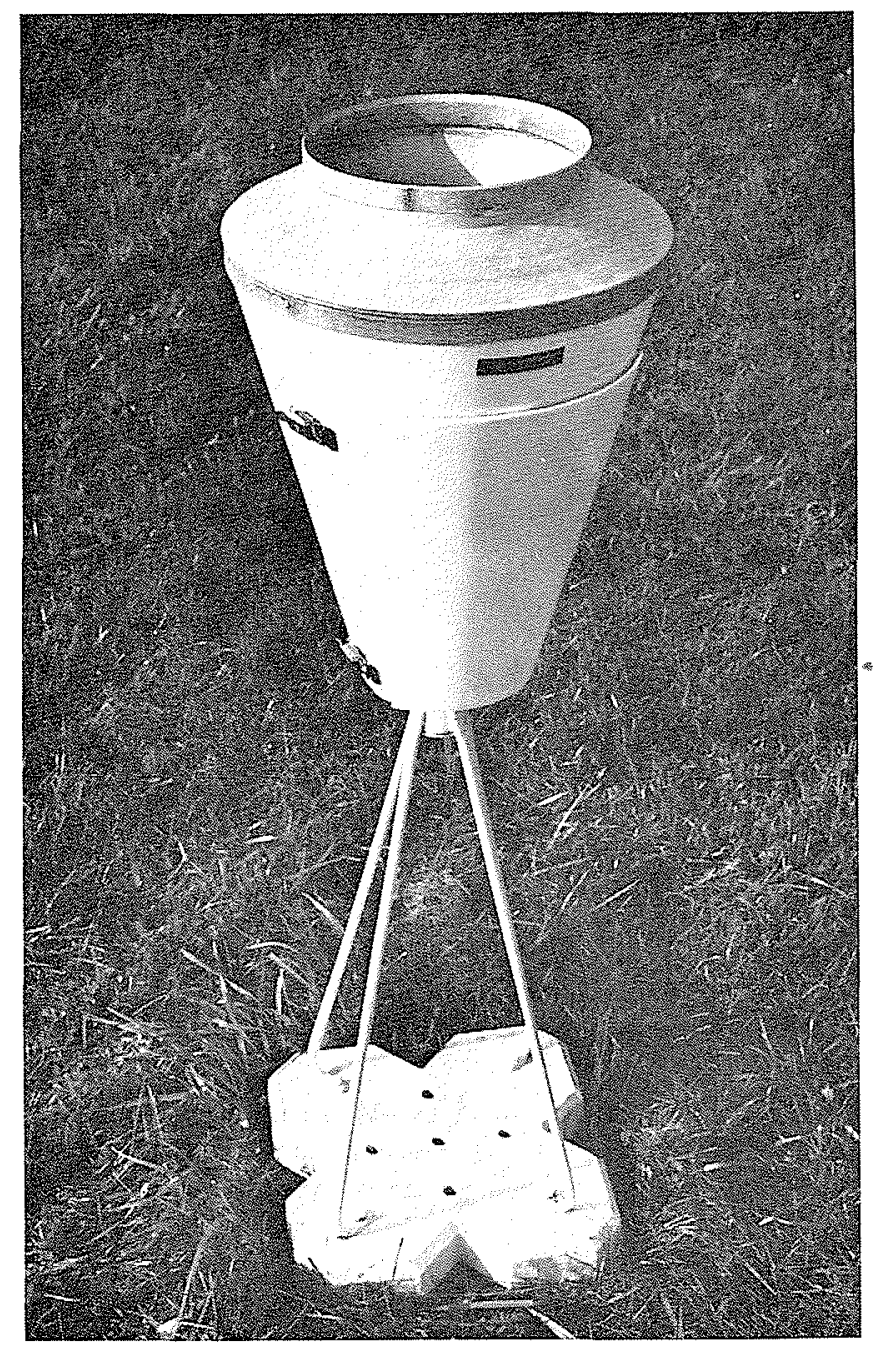

3/ Pluviographe type A

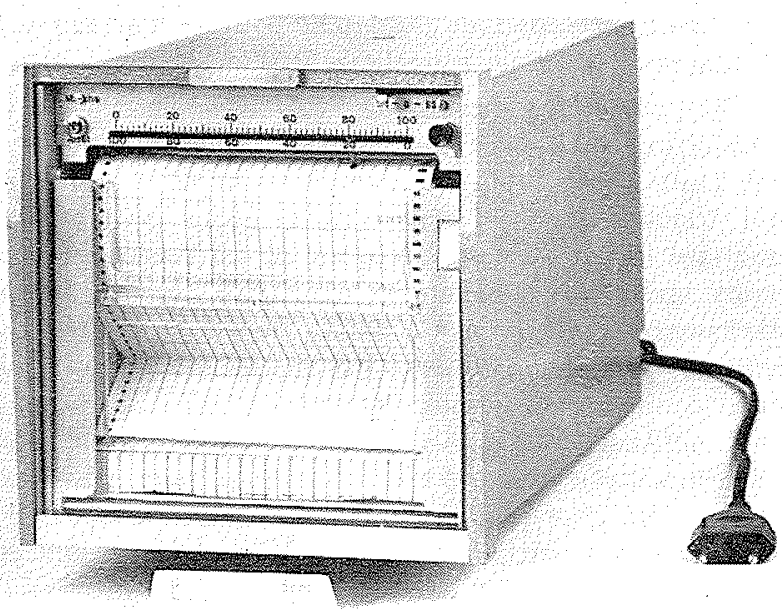

2/ Chronototalisateur.
Le démarrage sera d'autant plus précoce que l'intensité de la pluie frappant le déflecteur est plus importante. Cet aménagement a sensiblement amélioré les caractéristiques du compteur à augets pour les intensités supérieures à $150 \mathrm{~cm}^{3} / \mathrm{mn}$.

Pour des raisons de commodité de réglage et de mise en place, les augets et leur support ont été placés dans un petit coffret métallique de forme parallélépipédique fixé sur une platine dont on peut aisément régler l'horizontalité grâce à trois vis calantes.

Nous avons choisi pour ce pluviographe, une bague dont la surface est de $1000 \mathrm{~cm}^{2}$ et une capacité des augets de $20 \mathrm{~cm}^{3}$ conduisant à un incrément de mesure de $0,2 \mathrm{~mm}$. Ce choix peut apparaitre discutable aux utilisateurs des anciens pluviographes qui fournissaient un incrément de $0,1 \mathrm{~mm}$. Il résulte de considérations techniques et économiques.

L'utilisation d'une bague de $2000 \mathrm{~cm}^{2}$ n'a pas été retenue car elle conduisait à la réalisation d'un appareil trop volumineux, plus sensible aux effets du vent et pour lequel les 


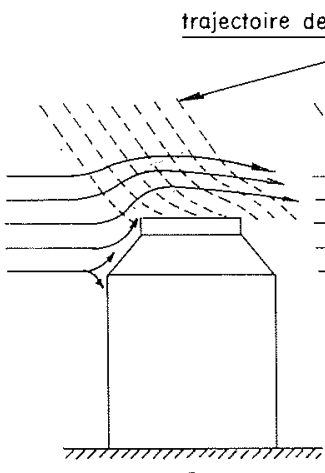

a

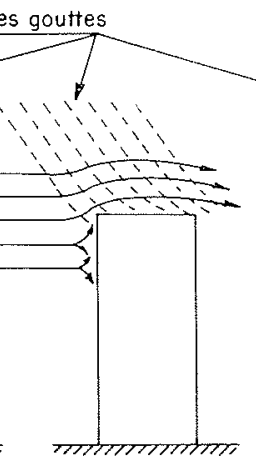

$\mathrm{b}$

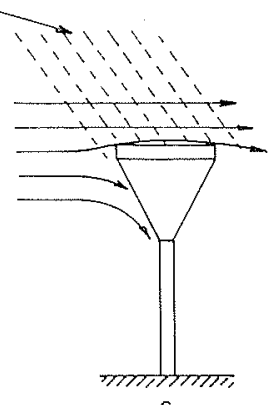

4/ Modification du champ des précipitations à l'avant et au-dessus des pluviomètres de forme différente.

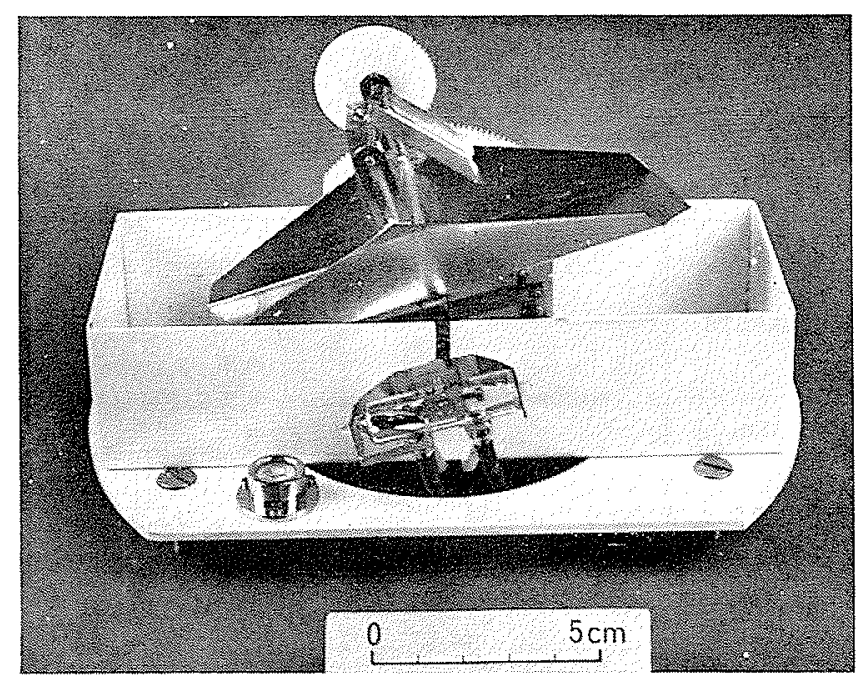

5/ Compteur volumétrique générateur d'impulsions.

pertes d'eau par évaporation et par rejaillissement auraient été plus importantes.

L'association d'un compteur à augets de $10 \mathrm{~cm}^{3}$ à la bague de $1000 \mathrm{~cm}^{2}$ a été envisagée puis écartée au profit du compteur à augets de $20 \mathrm{~cm}^{3}$ moins sensible que le précédent aux effets de l'intensité en raison de ses basculements plus rapides et deux fois moins fréquents.

Le générateur d'impulsions (figure 5) solidaire de l'étrier supportant les augets est constitué par un contacteur à mercure. A chaque basculement, il ferme un circuit électrique pendant un court instant.

Le carter a été aménagé de manière à ce qu'on puisse, sans la moindre modification, ajouter un dispositif de réchauffage capable de maintenir la température de la surface du cône récepteur à +4 "C lorsque la température extérieure varie de +4 à -10 " $\mathrm{C}$. Ce système comprend une résistance électrique répartie clans une pièce plaquée contre la face interne du cône et un thermostat de contact coupant l'alimentation électrique de la résistance pour des températures supérieures à $+4{ }^{\circ} \mathrm{C}$. La puissance dissipée est d'environ $230 \mathrm{~W}$ sous $220 \mathrm{~V}$.
Au-dessous de la platine supportant le compteur à augets, il reste un volume suffisant pour y placer, le cas échéant, un petit compteur numérique électromécanique et les piles nécessaires à son fonctionnement (figure 1).

Les impulsions issues du transducteur sont transmises au chronototalisateur par une liaison bifilaire. Le circuit de mesure qui comprend le câble et le contacteur lié aux augets, comporte au niveau de l'enregistreur, une alimentation de $12 \mathrm{~V}$ (constituée par 8 piles de $1,5 \mathrm{~V}$ type torche situées à l'arrière de l'appareil), un petit amplificateur de ligne et un électroaimant. A toute impulsion parcourant le circuit, l'électroaimant agit sur une roue à rochets et la fait avancer d'une dent. La rotation de cet élément provoque, par l'intermédiaire d'un système comprenant une came et divers leviers, un déplacement, correspondant à $0,2 \mathrm{~mm}$ de précipitation, du dispositif d'inscription perpendiculairement au sens du déroulement du diagramme. L'intérêt de l'amplificateur de ligne est de permettre des télémesures sur de grandes distances (jusqu'à $20 \mathrm{~km}$ avec des conducteurs téléphoniques de $0,3 \mathrm{~mm}^{2}$ de section).

Une prise située à l'arrière de l'appareil permet d'opérer un branchement sans piles sur alimentation extérieure (12 V).

La table de déroulement fonctionne avec un diagramme plié. Les vitesses de déroulement sont de 15 et $60 \mathrm{~mm} / \mathrm{h}$, avec possibilité de passer facilement de l'une à l'autre en manœuvrant un petit levier situé sur la face avant du coffret. La longueur du diagramme est $\mathrm{dz} 13 \mathrm{~m}$; elle autorise un enregistrement mensuel ou hebdomadaire suivant la vitesse de défilement adoptée. La largeur utile de $120 \mathrm{~mm}$ est divisée en 50 intervalles égaux représentant chacun $0,2 \mathrm{~mm}$ de pluie.

L'entraînement de la table de défilement peut être assuré de trois façons différentes:

1. par moteur synchrone 220 ou $110 \mathrm{~V} 50 \mathrm{~Hz}$.

2. par moteur pas à pas fonctionnant à partir d'une alimentation de $24 \mathrm{~V}$ à impulsions demi seconde alternées.

3. par moteur mécanique à remontage automatique par piles (les piles alimentent le générateur d'impulsions et assurent aussi le remontage du moteur mécanique).

Ces trois possibilités ont été prévues pour répondre aux éventualités suivantes:

- dans le cas des stations météorologiques déjà pourvues d'une base de temps fournissant des signaux convenables, un chronototalisateur muni d'un moteur pas à pas fournit un enregistrement des précipitations synchrone avec les autres enregistrements effectués à la station;

- dans les stations ne disposant pas de cette alimentation, mais possédant néanmoins une alimentation électrique secourue, l'entraînement du diagramme se fera à l'aide d'un moteur synchrone;

- enfin, dans les stations où l'on ne dispose pas d'énergie, le seul moyen d'effectuer un enregistrement permanent, est de munir le chronototalisateur d'un moteur mécanique.

Les trois moteurs sont interchangeables.

Pour permettre à l'observateur de totaliser la hauteur de précipitations en $1 / 10 \mathrm{de} \mathrm{mm}$ sur une période donnée, il existe la possibilité de brancher en parallèle sur le chrono- 
totalisateur un compteur électromécanique avec remise à zéro.

Pour faciliter la remise à zéro de l'enregistrement soit après les précipitations, soit à période fixe, on peut ajouter des impulsions par l'intermédiaire d'un bouton poussoir, situé sur la face avant.

Les performances et caractéristiques de l'ensemble pluviomètre transducteur d'impulsion - chronototalisateur peuvent se résumer ainsi :

- domaine de mesure jusqu'à $250 \mathrm{~mm} / \mathrm{h}$ sans accumulation d'eau dans le cône;

- l'enregistrement s'effectuant sur un diagramme à grille orthogonale, l'intensité représentée par la pente de la courbe est facilement repérable à l'aiđe d'abaques. Les intensités maximales commodément mesurables sont de $40 \mathrm{~mm} / \mathrm{h}$ et $160 \mathrm{~mm} / \mathrm{h}$ pour des vitesses de défilement de 15 et $60 \mathrm{~mm} / \mathrm{h}$;

- il est moins sensible aux effets de l'intensité que ne l'était l'ancien pluviographe (figure 6) comme l'atteste le tableau ci-dessous.

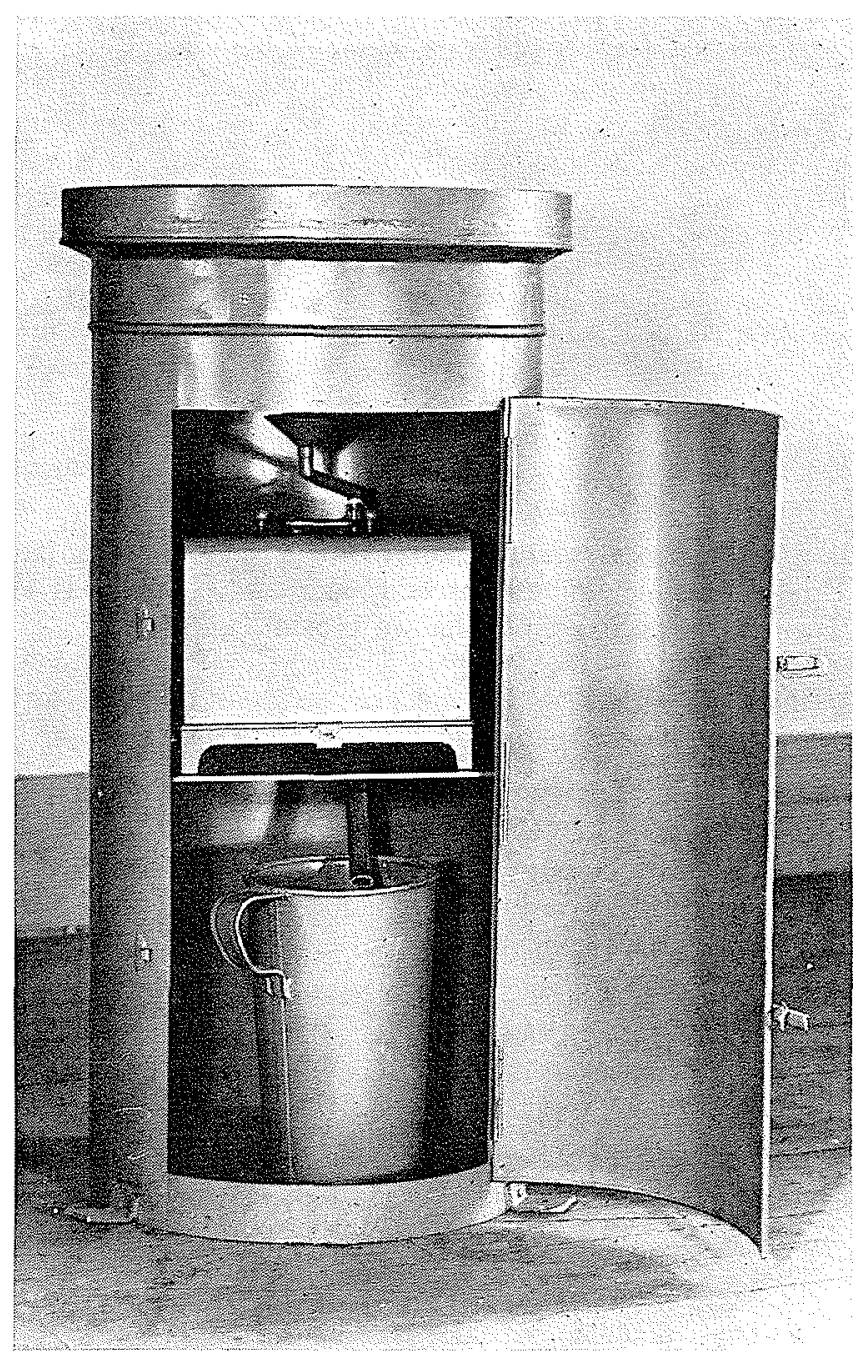

6/ Pluviographe ancien modèle.

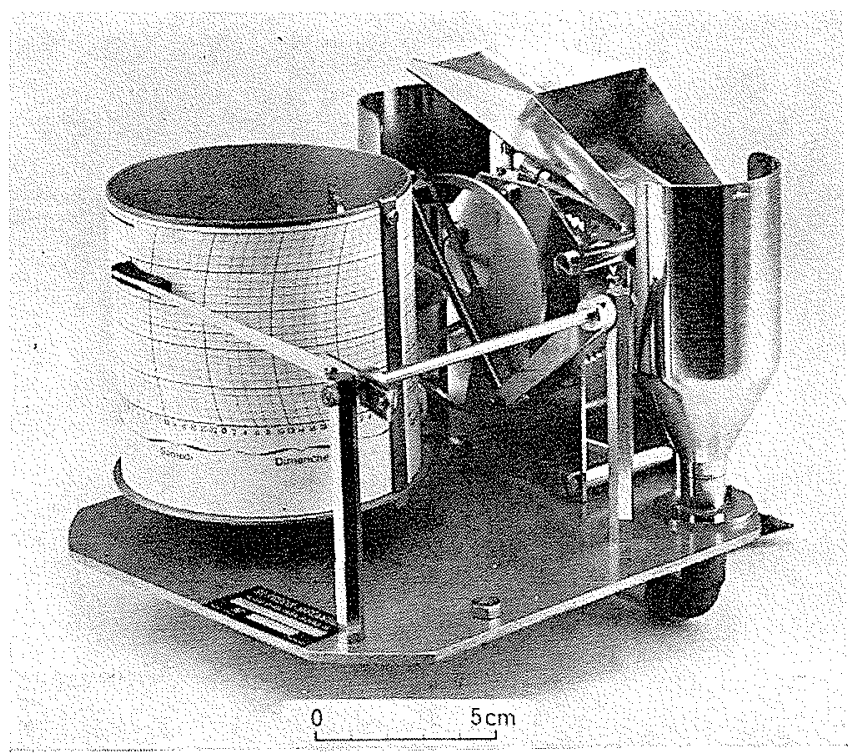

7/ Mécanisme d'enregistrement du pluviographe type A.

\begin{tabular}{|c|c|c|}
\hline \multirow[b]{2}{*}{$\begin{array}{l}\text { INTENSITÉ } \\
\text { (en } \mathrm{mm} / \mathrm{h} \text { ) }\end{array}$} & \multicolumn{2}{|c|}{ ERreur en $\%$ sur les QUANTtTÉS de Pluie } \\
\hline & $\begin{array}{l}\text { PLUVIOGRAPHE } \\
\text { NOUVEAU MODĖLEE }\end{array}$ & $\begin{array}{l}\text { Pluviographe } \\
\text { ANCIEN MODĖLE }\end{array}$ \\
\hline 75 & $\%$ & $12,5 \%$ \\
\hline 120 & $7 \%$ & $20 \%$ \\
\hline 180 & $9 \%$ & $>$ à 24 \\
\hline 250 & $10,5 \%$ & $>$ à $24 \%$ \\
\hline
\end{tabular}

Son dispositif de réchauffage a été prévu pour assurer la fusion de la neige dans des stations de moyenne et basse altitude; il ne peut convenir en haute montagne ou dans des lieux où règnent des conditions d'environnement particulièrement sévères. Dans ce dernier cas, seuls des appareils particulièrement étudiés peuvent fonctionner sans pour autant fournir une mesure représentative par suite de la récupération de quantités d'eau résultant de la fusion soit de givre, soit de la neige apportée dans le cône par le vent.

Une dernière caractéristique de cet équipement est sa commodité d'emploi. Grâce à l'enregistrement à distance dans la salle d'observations, l'observateur peut aisément surveiller l'évolution des précipitations. Son travail se trouve facilité par l'utilisation d'un diagramme plié qui lui permet à tout moment, sans interrompre l'inscription, de consulter l'enregistrement. Il est également possible de lire sur le compteur électromécanique associé la quantité d'eau recueillie depuis la dernière remise à zéro sans avoir à examiner le diagramme.

Le pluviographe type $A$ est un appareil plus simple que le précédent qui ne permet pas de faire des mesures de l'équivalent en eau de la neige ni d'effectuer un enregistrement à distance; le système de mesure et l'enregistrement sont situés dans le carter sous le cône de réception (figure 7). 
Les augets, d'une capacité de $20 \mathrm{~cm}^{3}$, ne sont pas munis de déflecteurs auxiliaires; ils agissent par l'intermédiaire d'une roue à rochets, sur un style inscripteur. Le déplacement du style s'effectue sur un diagramme curviligne entraîné par un cylindre dont la rotation est assurée par un mouvement d'horlogerie à remontage manuel.

Ce diagramme hebdomadaire a une largeur utile de $60 \mathrm{~mm}$, sa vitesse de déroulement est de $1,67 \mathrm{~mm} / \mathrm{h}$. La surface de la bague captrice étant de $400 \mathrm{~cm}^{2}$, l'incrément qui en résulte est de $0,5 \mathrm{~mm}$. Une largeur de diagramme correspondant à 50 basculements représente une hauteur de précipitation de $25 \mathrm{~mm}$.

La base du pied support est suffisamment pesante et large pour maintenir le pluviographe sans être obligé de le sceller dans le sol. Cette présentation a l'avantage de faciliter l'implantation, le déplacement et le réemploi de l'appareil.

Sur le plan des performances, des essais comparatifs ont montré que ce pluviographe fournissait des résultats avec une précision acceptable sur des quantités cumulées de précipitation. Les erreurs restent inférieures à $12 \%$ pour des intensités ne dépassant pas $150 \mathrm{~mm} / \mathrm{h}$. Dans le cas des faibles pluies (inférieures à quelques $\mathrm{mm}$ ) compte tenu de l'importance de l'incrément $(0,5 \mathrm{~mm})$ les indications fournies par l'appareil sont souvent erronées. Toutefois, cet inconvénient ne paraît pas gêner les utilisateurs qui ne s'intéressent qu'aux précipitations importantes.

La vitesse de défilement étant faible, il est impossible de déterminer l'intensité des précipitations, seules les périodes pendant lesquelles il pleut sont repérables.

Pour l'instant, les essais se poursuivent en effectuant de petites modifications de détail en vue d'optimaliser les possibilités du pluviographe tout en essayant de maintenir son prix au niveau actuel. Dès le milieu 1973, ce pluviographe devrait être opérationnel et commercialisé à un prix qui se situera autour de $1000 \mathrm{~F}$. 